On the Growth of the Ground-State Binding Energy with Increase in Potential Strength

\title{
Barry Simon
}

Citation: Journal of Mathematical Physics 10, 1415 (1969); doi: 10.1063/1.1664983

View online: http://dx.doi.org/10.1063/1.1664983

View Table of Contents: http://aip.scitation.org/toc/jmp/10/8

Published by the American Institute of Physics

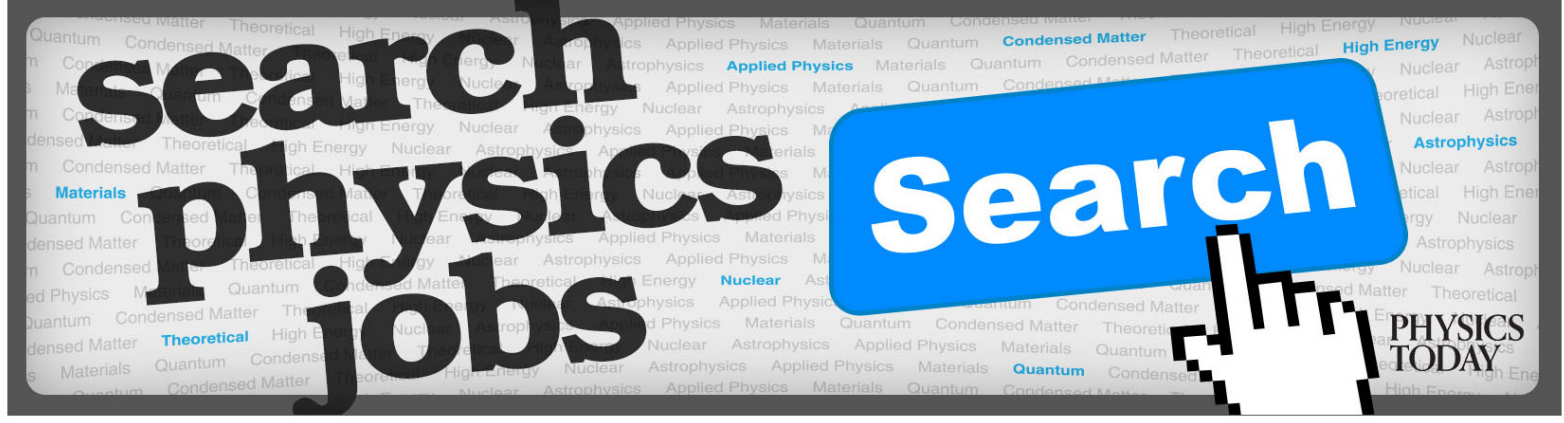




\title{
On the Growth of the Ground-State Binding Energy with Increase in Potential Strength*
}

\author{
BARRY SIMON $\dagger$ \\ Princeton University, Princeton, New Jersey
}

(Received 7 February 1969)

\begin{abstract}
We study the asymptotic behavior of the ground-state binding energy $G(\lambda)$ of $-\Delta+\lambda V$ as $\lambda \rightarrow \infty$. Unlike the number of bound states, $G(\lambda)$ does not have a universal power growth as $\lambda \rightarrow \infty$. It is shown, however, that as $\lambda \rightarrow \infty$ for Kato potentials

$$
A \lambda<G(\lambda)<B \lambda^{4} .
$$

Examples are presented for which $G \sim \lambda^{\beta}$ for any $1<\beta<4$. Other examples are presented which obey no power growth. We also prove theorems which reflect the close connection between the large $\lambda$ behavior of $G$ and the small $r$ behavior of $V$ for potentials with a single at tractive singularity at $r=0$. These can be roughly phrased as follows: If $V \sim-r^{-\alpha}$ for $\mathbf{r} \rightarrow 0$, then $G(\lambda) \sim \lambda^{\beta}$ with $\beta=2 /(2-\alpha)$ as $\lambda \rightarrow \infty$.
\end{abstract}

\section{INTRODUCTION}

For large classes of potentials it has been shown that the total number of bound states $[N(\lambda V)]$, the number of states in any $l$ channel $\left[n_{l}(\lambda V)\right]$, and the largest $l$-channel with bound states $\left[l_{\max }(\lambda V)\right]$, for the Hamiltonian $-\Delta+\lambda V$, all have power growth in $\lambda$ as $\lambda \rightarrow \infty$. Specifically,

$$
\begin{array}{cl}
A \lambda^{\frac{3}{2}}<N(\lambda V)<B \lambda^{\frac{3}{2}} & \text { (see Ref. 1), } \\
a \lambda^{\frac{1}{2}}<l_{\max }(\lambda V)<b \lambda^{\frac{1}{2}} & \text { (see Ref. 1), } \\
C_{l} \lambda^{\frac{1}{2}}<n_{l}(\lambda V)<D_{l} \lambda^{\frac{1}{2}} \quad \text { (see Ref. 2), }
\end{array}
$$

where each formula holds for $\lambda$ sufficiently large, and the constants are $V$-dependent. However, the powers $\frac{1}{2}$ or $\frac{3}{2}$ are not $V$-dependent.

In this paper, we examine the analogous question for $G(\lambda V)$, the binding energy of the ground state of $-\Delta+\lambda V$. We define $G$ so it is positive, i.e., $G$ is the negative of the energy of the bound state. We only deal with potentials which are "negative somewhere" (see Ref. 1, Corollary 1). In this case if $\lambda_{0}=$ $\inf \{\lambda>0 \mid N(\lambda V)>0\}, G(\lambda V)$ only makes sense if $\lambda>\lambda_{0}$, and so we henceforth suppose $\lambda>\lambda_{0}$.

We see that, unlike $N, n_{l}$, and $l_{\max }, G$ does not necessarily have power growth and, when it does, the power can vary between 1 and 4 ; i.e., there is not a universal power growth. We also show that, when $V$ is a (not necessarily central) potential with a single attractive singularity $\mathbf{r}=0$, that power growth of $V$ at $\mathbf{r}=0$ leads to power growth of $G$ as $\lambda \rightarrow \infty$. Thus, the large coupling-constant behavior of $G$

* This research partially sponsored under Air Force Office of Scientific Research under Contract AF49(638)-1545.

$\dagger$ N.S.F. pre-doctoral fellow.

1 B. Simon, J. Math. Phys. 10, 1123 (1969); F. Calogero and G. Cosenza, Nuovo Cimento 45A, 867 (1966).

${ }^{2}$ F. Calogero, Commun. Math. Phys. 1, 80 (1965); Variable Phase Approach to Potential Scattering (Academic Press Inc., New York, 1967), Chap. 23; J. H. E. Cohn, J. London Math. Soc. 40, 523 (1965); 41, 469 (1966); W. Frank, J. Math. Phys. 8, 466 (1967). "mirrors" the small $\mathbf{r}$ behavior of $V$, which is an intuitively expected result.

For convenience, we restrict ourselves to potentials $V$ which are the sum of an $L^{2}$ and an $L^{\infty}$ function. This class was first considered by Kato, ${ }^{3}$ who showed that $-\Delta+V$ is self-adjoint on $D(\Delta)$, the domain of $\Delta$. We call such potentials Kato potentials.

Let us summarize the remainder of the paper. In Sec. 2, we discuss various types of power growth. In Sec. 3, we present a class of Kato potentials for which $G(\lambda) \sim \lambda^{\beta}$ for any $1<\beta<4$. In Sec. 4 , we derive several general properties of $G$, including the fact ${ }^{4}$ that any power growth must have $1 \leq \beta \leq 4$. In Sec. 5, we show that, if $V$ has power growth $V \sim-r^{\alpha}$ for $r$ small $\left(0<\alpha<\frac{3}{2}\right)$, then $G \sim \lambda^{\beta}$ with $\beta=$ $2 /(2-\alpha)$. Finally, in Sec. 6 , we present a $V$ with $G(\lambda)$ not possessing power growth as $\lambda \rightarrow \infty$.

\section{TYPES OF POWER GROWTH}

For a function $F(\lambda)$, there are at least five natural interpretations for the expression $F(\lambda) \sim \lambda^{\alpha}$ :

(a) There is a $C$ such that, for any $\epsilon$, there is an $\Omega_{0}$, with

$(C-\epsilon) \lambda^{\alpha}<F(\lambda)<(C+\epsilon) \lambda^{\alpha}$, for $\lambda>\Omega_{0}$.

(b) There exist $C, C^{\prime}$, and $\Omega_{0}$, such that

$$
C \lambda^{\alpha}<F(\lambda)<C^{\prime} \lambda^{\alpha}, \text { for } \lambda>\Omega_{0} \text {. }
$$

(c) For every $\epsilon$, there is an $\Omega_{0}$ such that

$$
\lambda^{\alpha-\epsilon}<F(\lambda)<\lambda^{\alpha+\epsilon}, \text { for } \lambda>\Omega_{0} .
$$

(d) $\lim _{\lambda \rightarrow \infty}\left(\frac{\lambda}{F(\lambda)} \frac{\partial F}{\partial \lambda}\right)=\alpha$.

(e) $\lim _{\lambda \rightarrow \infty} \frac{F^{\prime}(\lambda)}{\lambda^{\alpha-1}}$ exists.

${ }^{3}$ T. Kato, Trans. Am. Math. Soc. 70, 195 (1951)

${ }^{4} \beta<4$ depends essentially on the fact that $V$ is Kato. If we are less restrictive and allow $V \sim r^{-\alpha}$ with $2>\alpha>\beta$, we get $G \sim \lambda \beta$ with $\beta>4$. 
(a), (b), and (c) can be rewritten in terms of limits:

(a) $\lim \left[F(\lambda) / \lambda^{\alpha}\right]$ exists and is finite and nonzero.

$\left(\mathrm{b}^{\prime}\right) \stackrel{\lambda \rightarrow \infty}{\lim }\left[F(\lambda) / \lambda^{\alpha}\right]$ and $\underline{\lim }\left[F(\lambda) / \lambda^{\alpha}\right]$ are finite and nonzero.

(c') $\lim _{\lambda \rightarrow \infty} \log F(\lambda) / \log \lambda=\alpha$.

We also remark that (c) is equivalent to a form with $C, C^{\prime}$ as in (b). The five types of growth are related by:

Theorem 2.1:

$$
\stackrel{\substack{\text { (a) } \rightarrow \text { (b) } \\ \text { (e) } \rightarrow \text { (d) } \rightarrow \text { (c) }}}{\searrow}
$$

and $(\mathrm{d})+(\mathrm{a}) \rightarrow(\mathrm{e})$. Moreover, no additional implications (single or multiple) hold.

Proof: $($ a $) \rightarrow($ b) $\rightarrow$ (c) is immediate, as is (d) + $\left(a^{\prime}\right) \rightarrow(e)$.

To see that $(d) \rightarrow(c)$, suppose (d) holds and $\epsilon$ is given. Then find $\Omega_{0}$ so that $\lambda>\Omega_{0}$ implies

$$
\frac{\alpha-\epsilon}{\lambda}<\frac{1}{F(\lambda)} \frac{\partial F}{\partial \lambda}<\frac{\alpha+\epsilon}{\lambda},
$$

which, integrated from $\Omega_{0}$ to $\lambda$ and then exponentiated, says

$$
\left(\frac{\lambda}{\Omega_{0}}\right)^{\alpha-\epsilon}<\frac{F(\lambda)}{F\left(\Omega_{0}\right)}<\left(\frac{\lambda}{\Omega_{0}}\right)^{\alpha+\epsilon}
$$

which is (c) (in the $C, C^{\prime}$ form).

(e) $\rightarrow$ (a) requires the integration of an inequality as in (d) $\rightarrow$ (c). This integration also shows that when (e) holds,

$$
\lim \frac{F^{\prime}(\lambda)}{\lambda^{\alpha-1}}=\alpha \lim \frac{F(\lambda)}{\lambda^{\alpha}},
$$

which is L'Hôpital's rule. This means that $(e) \rightarrow(d)$.

To see that no other implications hold, we remark that $F(\lambda)=\lambda\left(2+\sin \lambda^{-1}\right)$ obeys (b) and (d) but not (a) or (e); $F(\lambda)=\lambda \log \lambda$ obeys (c) but not (a), (b), or (e); and $F(\lambda)=\left[1+(1+\lambda)^{-1} \sin \lambda^{4}\right]$ obeys (a), (b), (c) but not (d) or (e).

Q.E.D.

We write $F(\lambda) \sim \lambda^{\alpha}(-)$, to indicate growth of type $(-)$. We remark that similar power growth for $V(r)$ at $r \rightarrow 0$ is also meaningful. In this notation, we have the following:

Theorem 2.2: For a large class of potentials, $N(\lambda V) \sim \lambda^{\frac{3}{2}}(\mathrm{~b}) ; n_{l}(\lambda V) \sim \lambda^{\frac{1}{2}}(\mathrm{~b}) ; l_{\max }(\lambda V) \sim \lambda^{\frac{1}{2}}(\mathrm{~b})$. (See Ref. 5.) For proof, see Refs. 1 and 2.

\footnotetext{
${ }^{5}$ K. Chadan [Nuovo Cimento S8A, 191 (1968)] has shown that
} $n_{l}(\lambda V) \sim \lambda^{\frac{1}{2}}$ (a) for a restricted set of $V$.

\section{A CLASS OF EXAMPLES WITH DIFFERENT POWER GROWTH}

Consider the class of potentials, $V_{\alpha}(r)=-r^{-\alpha}$. For $V$ to be Kato, we must ${ }^{6}$ have $0<\alpha<\frac{3}{2}$. We first remark that $-\Delta+\lambda V_{\alpha}$ always has bound states; in fact, because $V$ falls at $\infty$ more slowly than $r^{-2}$, $-\Delta+\lambda V$ always has infinitely many bound states ${ }^{7}$; we do, in fact, prove this below.

Theorem 3.1: There is a unitary transformation $U(\lambda, \alpha)$ so that

$$
U(\lambda, \alpha)^{-1}\left(-\Delta+\lambda V_{\alpha}\right) U(\lambda, \alpha)=\lambda^{\beta}\left(-\Delta+V_{\alpha}\right),
$$

where $\beta=2 /(2-\alpha)$.

Proof: The formal scaling $\mathbf{r} \rightarrow \lambda^{\beta / 2} \mathbf{r}$ takes $-\Delta+\lambda V_{\alpha}$ to $\lambda^{\beta}\left(-\Delta+V_{\alpha}\right)$. Thus we let

$$
[U(\lambda, \alpha) \psi](\mathbf{r})=\lambda^{3 \beta / 4} \psi\left(\lambda^{\beta / 2} \mathbf{r}\right) .
$$

Then $U$ is unitary and $U^{-1}(\Delta) U=\lambda^{\beta} \Delta, U^{-1} V U=$ $\lambda^{\alpha \beta / 2} V$. But

$$
\frac{1}{2} \alpha \beta+1=1+[\alpha /(2-\alpha)]=2 /(2-\alpha)=\beta,
$$

so the theorem is proven.

Corollary 3.2: For any $\lambda_{1},-\Delta+\lambda_{1} V_{\alpha}$ has infinitely many bound states.

Proof: A simple variational-principle argument as in Simon, ${ }^{1}$ Corollary 5 , shows that $-\Delta+\lambda V_{\alpha}$ has arbitrarily many bound states for $\lambda$ sufficiently large [i.e., $N(\lambda V) \rightarrow \infty$ ]. But by Theorem 3.1, $-\Delta+\lambda V_{\alpha}$ and $-\Delta+\lambda_{1} V_{\alpha}$ are unitarily equivalent up to a factor and so have the same number of bound states.

Corollary 3.3: $G\left(\lambda V_{\alpha}\right)=\lambda^{\beta} G\left(V_{\alpha}\right)$, and thus

$$
G\left(\lambda V_{\alpha}\right) \sim \lambda^{\beta}(\mathrm{e})
$$

Thus, in particular, any power growth $1<\beta<4$ is possible for Kato potentials. We can see that $\beta=1$ and $\beta=4$ are also possible. [For $\beta=1$, see Theorem 5.1 ; for $\beta=4$, we remark that Theorem 5.3(iii) implies that $V(r)=r^{-\frac{3}{2}}(1+|\log r|)^{-1}$, which is Kato, has $G(\lambda V) \sim \lambda^{4}(\mathrm{c})$.]

\section{GENERAL PROPERTIES OF $G(V)$}

Let us write $G(\lambda)$ for $G(\lambda V)$ when $V$ is held fixed.

Theorem 4.1: There is an $\Omega$ and a $B>0$ such that $G(\lambda)>B \lambda$ if $\lambda>\Omega$.

\footnotetext{
- Physically we expect no trouble if $\alpha<2$, but the mathematics is more complicated for $\alpha>\frac{3}{2}$, so we restrict ourselves.

${ }^{2}$ R. Courant and D. Hilbert, Methods of Mathematical Physics (Interscience Publishers, Inc., New York, 1953), Vol. I, p. 447.
} 
Proof: Let $\lambda_{0}$ be as in Sec. 1 and let $\psi$ be the ground state of $-\Delta+\left(\lambda_{0}+1\right) V$. Then $b=\langle\psi, V \psi\rangle\langle 0$, so that $\langle\psi,(-\Delta+\lambda V) \psi\rangle=a+\lambda b$ with $b<0$. By the variational principle, $G(\lambda)>-a-\lambda b$. Let $\Omega=$ $-2 a / b, B=-b / 2$, and the theorem follows.

Theorem 4.2: There is a $c>0$ such that $G(\lambda)<$ $C \lambda^{4}$ for $\lambda$ sufficiently large. ${ }^{4}$

Proof $^{8}$ : First suppose $V \in L^{2}$. Let $\psi_{\lambda}$ represent the ground state, and write $k^{2}=G(\lambda)$, with $k>0$. By Kato's theorem, $\psi_{\lambda} \in D(\Delta)$ so that $(-\Delta+\lambda V) \psi_{\lambda}=$ $-k^{2} \psi_{\lambda}$ implies $\psi_{\lambda}=\left(\Delta-k^{2}\right)^{-1}(\lambda V) \psi_{\lambda}$. Thus $\psi_{\lambda}$ obeys the integral equation

$$
\psi_{\lambda}(x)=-\frac{\lambda}{4 \pi} \int \frac{\exp (-k|x-y|)}{|x-y|} V(y) \psi_{\lambda}(y) d^{3} y .
$$

Thus

$$
\begin{aligned}
\int d^{3} x & \left|\psi_{\lambda}(x)\right|^{2} \\
< & \frac{\lambda^{2}}{(4 \pi)^{2}} \int d^{3} x \int d^{3} y \int d^{3} y^{\prime}\left|\psi_{\lambda}(y)\right|\left|\psi_{\lambda}\left(y^{\prime}\right)\right| \\
& \times \frac{|V(y)|\left|V\left(y^{\prime}\right)\right| \exp \left[-k\left(|x-y|+\left|x-y^{\prime}\right|\right)\right]}{|x-y|\left|x-y^{\prime}\right|} \\
= & \frac{\lambda^{2}}{(4 \pi)^{2}} \int d^{3} y \int d^{3} y^{\prime}|V(y)|\left|V\left(y^{\prime}\right)\right|\left|\psi_{\lambda}(y)\right|\left|\psi_{\lambda}\left(y^{\prime}\right)\right| \\
& \times \int d^{3} x \frac{\exp \left[-k\left(|x-y|+\left|x-y^{\prime}\right|\right)\right]}{|x-y|\left|x^{\prime}-y\right|} .
\end{aligned}
$$

The $x$ integral is $(2 \pi / k) \exp \left(-k\left|y-y^{\prime}\right|\right) \leq 2 \pi / k .^{9}$ Thus,

$$
\begin{aligned}
\left\|\psi_{\lambda}\right\|^{2} & \leq \frac{\lambda^{2}}{8 \pi k}\left[\int d^{3} y|V(y)|\left|\psi_{\lambda}(y)\right|\right]^{2} \\
& \leq \frac{\lambda^{2}}{8 \pi k}\left\|\psi_{\lambda}\right\|^{2} \int d^{3} y|V(y)|^{2},
\end{aligned}
$$

where this last inequality follows from the CauchySchwartz theorem. Therefore, $k<C^{\frac{1}{2}} \lambda^{2}$ or $G(\lambda)=$ $k^{2}<C \lambda^{4}$.

Now suppose that $V=V_{1}+V_{2}$ with $V_{1} \in L^{2}$ and $V_{2} \in L^{\infty}$ and let $\left\|V_{2}\right\|_{\infty}=D$. Then $V>W$, where $W=V_{1}-D$, so $G(\lambda V)<G(\lambda W)=G\left(\lambda V_{1}\right)+\lambda D<$ $\lambda^{4} C+\lambda D$, and so the theorem holds true in this case.

Q.E.D.

Let us henceforth restrict ourselves to $V$ 's which lead to nondegenerate ground states for $-\Delta+\lambda V$; in particular, $V$ can be any central potential and any but the most pathological noncentral potential.

\footnotetext{
This is a modification of an argument, due to G. Tiktopoulos, private communication.

This can be done most easily in prolate spheroidal coordinates or by using the Green's-function equation for $\exp (-k|x-y|)$ / $|x-y|$.
}

Theorem 4.3: $G(\lambda)$ is a real analytic function for $\lambda>\lambda_{0}$ and $G^{\prime}(\lambda)=-\left\langle\psi_{\lambda}, V \psi_{\lambda}\right\rangle$, where $\psi_{\lambda}$ is the ground state.

Proof: $V$ is a small perturbation of $-\Delta+\lambda V$ in the technical sense. ${ }^{10}$ Since the ground state is nondegenerate, Rayleigh-Schrödinger perturbation theory for $G\left(\lambda+\lambda_{0}\right)$ (expanded in $\lambda$ ) converges, ${ }^{11}$ and so $G$ is real analytic. Moreover, $G^{\prime}(\lambda)$ is given by the perturbation theoretic result as $-\left\langle\psi_{\lambda}, V \psi_{\lambda}\right\rangle$. Q.E.D.

The condition $-G^{\prime}(\lambda)=\left\langle\psi_{\lambda}, V \psi_{\lambda}\right\rangle$ is the famous Feynman-Hellman theorem, ${ }^{12}$ whose proof is seen to be completely rigorous for Kato potentials.

\section{Theorem 4.4:}

(i) $G(\lambda)>0$;

(ii) $G^{\prime}(\lambda)>\lambda^{-1} G(\lambda)$

(iii) $G^{\prime \prime}(\lambda)>0$.

Proof:

(i) is immediate.

(ii) follows from the fact that $-\Delta$ is positive for

$$
G(\lambda)=\left\langle\psi_{\lambda}, \Delta \psi_{\lambda}\right\rangle-\lambda\left\langle\psi_{\lambda}, V \psi_{\lambda}\right\rangle<-\lambda G^{\prime}(\lambda) .
$$

(iii) follows from the fact that the second-order perturbation term for $-G(\lambda)$ is negative. Alternately, we can prove that $G^{\prime}(\lambda)$ is increasing directly, as follows: Let $\lambda_{1}>\lambda_{2}$ and write $\psi_{i}=\psi_{\lambda_{i}}$. By the variational principle,

$$
\begin{aligned}
\left.G\left(\lambda_{1}\right)=\left\langle\psi_{1}, \Delta \psi_{1}\right\rangle-\lambda_{1}\left\langle\psi_{1}, V \psi_{1}\right\rangle\right\rangle & \left\langle\psi_{2}, \Delta \psi_{2}\right\rangle \\
& -\lambda_{1}\left\langle\psi_{2}, V \psi_{2}\right\rangle, \\
\left.G\left(\lambda_{2}\right)=\left\langle\psi_{2}, \Delta \psi_{2}\right\rangle-\lambda_{2}\left\langle\psi_{2}, V \psi_{2}\right\rangle\right\rangle\left\langle\psi_{1}, \Delta \psi_{1}\right\rangle & -\lambda_{2}\left\langle\psi_{1}, V \psi_{1}\right\rangle .
\end{aligned}
$$

Adding, we see that

$$
\left(\lambda_{1}-\lambda_{2}\right)\left\langle\psi_{2}, V \psi_{2}\right\rangle>\left(\lambda_{1}-\lambda_{2}\right)\left\langle\psi_{1}, V \psi_{1}\right\rangle
$$

or

$$
-G^{\prime}\left(\lambda_{2}\right)>-G^{\prime}\left(\lambda_{1}\right) \text { Q.E.D. }
$$

(ii) can be used to give an alternate proof of Theorem 4.1. (iii) tells us that $G$ is convex. We remark that the reasonable conjecture that a smooth convex function has power growth is false, as our example in Sec. 6 shows.

10 T. Kato, Perturbation Theory for Linear Operators (SpringerVerlag, Berlin, 1966), pp. 375-377.

11 Reference 10 , pp. $381-382$

12 R. P. Feynman, Phys. Rev. 56, 340 (1939); H. Hellman, Einführung in die Quantenchemie (Franz Deuticke, Leipzig, 1937), p. 285 . 


\section{Theorem 4.5:}

(i) $G(\lambda)<-\left\langle\psi_{\lambda},\left(\lambda V+4 r^{-2}\right) \psi_{\lambda}\right\rangle$.

(ii) If $\partial V / \partial r$ exists and the Virial theorem is obeyed, then

$$
G(\lambda)=-\lambda\left\langle\psi_{\lambda}, \frac{1}{2} r(\partial V / \partial r)+V \psi_{\lambda}\right\rangle
$$

Proof:

(i) follows from the simple fact that, for any $\psi \in D(\Delta)$,

$$
\langle\psi,-\Delta \psi\rangle\rangle\left\langle\psi, \frac{1}{4 r^{2}} \psi\right\rangle
$$

(see Ref. 13 for a proof when $\psi$ is $C^{\infty}$ of compact support and then use the fact that $\Delta$ is the closure of its restriction to these functions).

(ii) follows from the Virial theorem

$$
2\left\langle\psi_{\lambda},-\Delta \psi_{\lambda}\right\rangle=\left\langle\psi_{\lambda}, r(\partial V / \partial r) \psi_{\lambda}\right\rangle \text {. Q.E.D. }
$$

For a $C^{1}$ central Kato potential, the standard proof of the Virial theorem ${ }^{14}$ should go through. For the noncentral case, Weidmann ${ }^{15}$ has given simple conditions for the Virial theorem to hold as a rigorous result.

Theorem 4.6: If $V_{1}$ and $V_{2}$ are Kato and $V_{1}(r)<$ $V_{2}(r)$ for all $r$, then $G\left(\lambda V_{1}\right)>G\left(\lambda V_{2}\right)$ (even if the $V_{i}$ do not go to 0 at $r=\infty$ ).

Proof: This is an immediate consequence of the variational principle. We have, in fact, already used this argument in the proof of Theorem 4.2.

\section{POWER GROWTH AT $r=0$}

Let us first eliminate the trivial case of potentials that are bounded below.

Theorem 5.1: If $V(r)>-C$ for some constant $C>0$, then $G(\lambda) \sim \lambda(\mathrm{e})$.

Proof: [Notice that (b) growth is trivial since we have Theorem 4.1 and $G(\lambda)<\lambda C$.] We have $G^{\prime}(\lambda)=$ $-\left\langle\psi_{\lambda}, V \psi_{\lambda}\right\rangle\left\langle C\right.$. Thus $G^{\prime}$ is bounded above, but $G^{\prime}$ is increasing by Theorem 4,4(iii). Thus $\lim G^{\prime}(\lambda)$ exists and is finite, i.e., $G(\lambda) \sim \lambda(\mathrm{e})$.

Q.E.D.

We deal in the remainder of this section with the more interesting case of potentials with attractive centers. We could deal with the case of finitely many centers, but we restrict ourselves to the following class.

\footnotetext{
13 Reference 7, p. 446 .
14 The normal proof really supposes $\psi_{\lambda} \in D\left[\left(\partial^{2} / \partial r^{2}\right) r(\partial / \partial r)\right]$. For negative energy eigenfunctions in a $C^{\mathbf{1}} \mathrm{K}$ ato potential, one should be able to prove that $\psi \lambda$ decreases exponentially from its integral equation. For the radial Schrödinger equation, it follows that $\psi_{\lambda}$ is $C^{3}$.

15 J. Weidmann, Bull. Am. Math. Soc. 73, 452 (1967).
}

Definition: We say a potential $V$ is attractive if

(i) $\lim _{r \rightarrow 0} V(\mathbf{r})=-\infty$,

(ii) for every $R>0, V$ is bounded in the region $\{\mathbf{r} \mid r>R\}$. (We say that $V$ is "bounded outside spheres.")

Definition: We say $V$ has attractive growth $\alpha(a)$ [or $\alpha(b), \alpha(c)$ ] if

(i) $V$ is attractive,

(ii) $V \sim-r^{-\alpha}$ (a) as $r \rightarrow 0$ [or (b) or (c)].

We reserve $\alpha(d)$ and $\alpha(e)$ for a slightly stronger condition.

We are heading towards proving that whenever $V$ has attractive growth $\alpha$ of some type, $G(\lambda) \sim \lambda^{\beta}$ with the same type of growth where $\beta=2 /(2-\alpha)$ : For growth of type (a), (b), or (c), the crucial element of the proof is the following:

Lemma 5.2: If $V(r)<-A r^{-\gamma}+B$, then $G(\lambda)>$ $A R_{\gamma} \lambda^{\delta}-B \lambda$; and if $V(r)>-C r^{-\gamma}-D$, then $G(\lambda)<$ $C R_{\gamma} \lambda^{\delta}+D \lambda$, where $\delta=2 /(2-\gamma)$, and $R_{\gamma}=G\left(V_{\gamma}\right)$ as in Sec. 3.

Proof: This follows immediately from Theorems 3.3 and 4.6 .

Theorem 5.3: Let $0<\alpha<\frac{3}{2}$. Then

(i) If $V$ has attractive growth $\alpha(a)$, then $G(\lambda) \sim$ $\lambda^{\beta}(\mathrm{a})$

(ii) If $V$ has attractive growth $\alpha(\mathrm{b})$, then $G(\lambda) \sim$ $\lambda^{\beta}(\mathrm{b})$.

(iii) If $V$ has attractive growth $\alpha(\mathrm{c})$, then $G(\lambda) \sim$ $\lambda^{\beta}(\mathrm{c})$.

In each case, $\beta=2 /(2-\alpha)$. In case (i),

$$
\lim _{\lambda \rightarrow \infty} \frac{G(\lambda)}{\lambda^{\beta}}=R_{\alpha} \lim _{r \rightarrow 0}-\frac{V(r)}{r^{\alpha}} .
$$

Proof: (i) Since $V$ is bounded outside spheres and $V \sim-r^{-\alpha}(\mathrm{a})$, there is a $C=\lim _{r \rightarrow \infty}-V(r) / r^{\alpha}$, so that for every $\epsilon$, there is a $B$ with

$$
-(C+\epsilon) r^{-\alpha}-B<V(r)<-(C-\epsilon) r^{-\alpha}+B .
$$

Thus Lemma 5.2 implies

$$
(C-\epsilon) R_{\alpha} \lambda^{\beta}-B \lambda<G(\lambda)<(C+\epsilon) R_{\alpha} \lambda^{\beta}+B \lambda .
$$

Since $\beta>1$, for every $\epsilon$ we have

$$
(C-\epsilon) R_{\alpha}<\underline{\lim } \frac{G(\lambda)}{\lambda^{\beta}}<\varlimsup \frac{G(\lambda)}{\lambda^{\beta}}<(C+\epsilon) R_{\alpha} .
$$

Since $\epsilon$ is arbitrary,

$$
\lim _{\lambda \rightarrow \infty} \frac{G(\lambda)}{\lambda^{\beta}}=R_{\alpha} C .
$$


(ii) Since $V$ is bounded outside spheres and $V \sim-r^{-\alpha}(\mathrm{b})$, there is a $B, C, C^{\prime}$ so that $-C r^{-\alpha}-$ $B<V(r)<-C^{\prime} r^{-\alpha}+B$. Thus Lemma 5.2 implies

$$
R_{\alpha} C^{\prime} \lambda^{\beta}-B \lambda<G(\lambda)<R_{\alpha} C \lambda^{\beta}+B \lambda .
$$

Thus for $\lambda$ sufficiently large,

$$
\frac{1}{2} R_{\alpha} C^{\prime} \lambda^{\beta}<G(\lambda)<2 R_{\alpha} C \lambda^{\beta} .
$$

(iii) Since $V$ is bounded outside spheres and $V \sim-r^{-\alpha}(\mathrm{c})$, for every $\epsilon$, there is a $B$ so that

$$
-r^{\alpha+\epsilon}-B \leq V(r)<-r^{\alpha-\epsilon}+B .
$$

Thus Lemma 5.2 implies

$$
-B \lambda+R_{\alpha-\epsilon} \lambda^{\beta-\epsilon^{\prime}} \leq G(\lambda) \leq R_{\alpha+\epsilon} \lambda^{\beta+\epsilon^{\prime \prime}}+B \lambda,
$$

so that

$$
\beta-\epsilon^{\prime}<\underline{\lim } \frac{\log G(\lambda)}{\log \lambda}<\varlimsup \frac{\log G(\lambda)}{\log \lambda}<\beta+\epsilon^{\prime \prime} .
$$

Since $\epsilon$ is arbitrary and $\epsilon^{\prime}, \epsilon^{\prime \prime} \rightarrow 0$ as $\epsilon \rightarrow 0$, we have

$$
\lim _{\lambda \rightarrow \infty} \frac{\log G(\lambda)}{\log \lambda}=\beta \text {. }
$$

Q.E.D.

We finally get to the interesting cases of Type (d) or (e) growth. The Virial theorem is crucial and so we restrict ourselves to the following:

Definition: We say $V$ has attractive growth $\alpha(\mathrm{d})$ or $\alpha(\mathrm{e})$ if

(i) $V$ is attractive,

(ii) $V \sim-r^{-\alpha}$ (d) [or (e)],

(iii) $V$ obeys a Virial theorem,

(iv) $r(\partial V / \partial r)$ is bounded outside spheres.

Condition (iv) is not essential in its strong form. Using the fact that $\psi_{\lambda}$ falls off exponentially, we could probably survive with $e^{-\alpha r}[r(\partial V / \partial r)]$ bounded for some $\alpha$. However, only the most pathological $V$ 's fail to obey (iv) and so we do not consider weakening it in detail.

Lemma 5.4: Let $f_{i}(\lambda), g_{i}(\lambda)(i=1,2)$ be positive functions with $g_{2} \rightarrow \infty$ as $\lambda \rightarrow \infty$. Suppose that

(i) $\varlimsup_{\lambda \rightarrow \infty} \frac{g_{1}}{g_{2}}=C<\infty$,

(ii) $\left|f_{i}(\lambda)-g_{i}(\lambda)\right| \leq B$ for some $B$ and all $\lambda$.

Then

$$
\underline{\lim } \frac{f_{1}(\lambda)}{f_{2}(\lambda)}=\underline{\lim } \frac{g_{1}(\lambda)}{g_{2}(\lambda)} ; \varlimsup \frac{f_{1}(\lambda)}{f_{2}(\lambda)}=\varlimsup \frac{g_{1}(\lambda)}{g_{2}(\lambda)} .
$$

Proof: We first remark that $f_{2} \rightarrow \infty$ as $\lambda \rightarrow \infty$ by (i) and (ii). Moreover,

$$
\begin{aligned}
\frac{f_{1}}{f_{2}}-\frac{g_{1}}{g_{2}} & =\frac{f_{1} g_{2}-f_{2} g_{1}}{f_{2} g_{2}}=\frac{\left(f_{1}-g_{1}\right) g_{2}-g_{1}\left(f_{2}-g_{2}\right)}{g_{2} f_{2}} \\
& =\frac{\left(f_{1}-g_{1}\right)}{f_{2}}-\frac{g_{1}}{g_{2}} \frac{\left(f_{2}-g_{2}\right)}{f_{2}} .
\end{aligned}
$$

But for $\lambda$ sufficiently large, $\left(g_{1} / g_{2}\right) \leq 2 C$, so

Thus

$$
\left|\frac{f_{1}}{f_{2}}-\frac{g_{1}}{g_{2}}\right| \leq \frac{B}{f_{2}}+\frac{2 C B}{f_{2}} \rightarrow 0 .
$$

$$
\lim _{\lambda \rightarrow \infty}\left|\frac{f_{1}}{f_{2}}-\frac{g_{1}}{g_{2}}\right|=0,
$$

so that the theorem follows.

Theorem 5.5: Let $0<\alpha<\frac{3}{2}$. Then:

(i) If $V$ has attractive growth $\alpha(\mathrm{d})$, then $G(\lambda) \sim$ $\lambda^{\beta}(\mathrm{d})$.

(ii) If $V$ has attractive growth $\alpha(\mathrm{e})$, then $G(\lambda) \sim$ $\lambda^{\beta}(\mathrm{e})$.

In either case, $\beta=2 /(2-\alpha)$. In case (ii),

$$
\lim _{\lambda \rightarrow \infty} \frac{G^{\prime}(\lambda)}{\lambda^{\beta-1}}=\frac{\beta R_{\alpha}}{\alpha} \lim _{r \rightarrow 0}-\frac{V^{\prime}(r)}{r^{\alpha-1}} .
$$

Proof: (i) By the Virial and Feynman-Hellman theorems,

$$
\frac{G(\lambda)}{\lambda G^{\prime}(\lambda)}=\frac{\left\langle\psi_{\lambda},\left(\frac{1}{2} r(\partial V / \partial r)+V\right) \psi_{\lambda}\right\rangle}{\left\langle\psi_{\lambda}, V \psi_{\lambda}\right\rangle} .
$$

Let us fix some $R$ and let

$$
\langle\psi, \phi\rangle_{R}=\int_{|\mathbf{r}|<R} d^{3} r \bar{\psi}(\mathbf{r}) \phi(\mathbf{r}) .
$$

Then $\left|\left\langle\psi_{\lambda}, V \psi_{\lambda}\right\rangle-\left\langle\psi_{\lambda}, V \psi_{\lambda}\right\rangle_{R}\right|$ and

$$
\begin{aligned}
\mid\left\langle\psi_{\lambda},\left(\frac{1}{2} r(\partial V / \partial r)+V\right) \psi_{\lambda}\right\rangle & \\
& -\left\langle\psi_{\lambda},\left(\frac{1}{2} r(\partial V / \partial r)+V\right) \psi_{\lambda}\right\rangle_{R} \mid
\end{aligned}
$$

are bounded,

$$
\varlimsup \frac{G(\lambda)}{\lambda G^{\prime}(\lambda)} \leq 1
$$

[by Theorem 4.4(ii)] and $\left\langle\psi_{\lambda}, V \psi_{\lambda}\right\rangle \rightarrow \infty$. Thus, by Lemma 5.4, the $\left\lceil\right.$ im and $\underline{\lim }$ of $G(\lambda) / \lambda G^{\prime}(\lambda)$ are the same as that of

$$
\frac{\left\langle\psi_{\lambda},\left(\frac{1}{2} r(\partial V / \partial r)+V\right) \psi_{\lambda}\right\rangle_{R}}{\left\langle\psi_{\lambda}, V \psi_{\lambda}\right\rangle_{R}}
$$

for any fixed $R$. Since $V(r) \rightarrow-\infty$, pick $R_{0}$, so that $V(\mathbf{r}) \leq-1$ for $|\mathbf{r}| \leq R_{\mathbf{0}}$. Given $\epsilon$, choose $R$ so that $R \leq R_{0}$ and

$$
\left|\frac{r}{V} \frac{\partial V}{\partial r}+\alpha\right| \leq \epsilon \text { for }|r| \leq R .
$$


Thus

$$
\begin{aligned}
&\left\langle\left\langle\psi_{\lambda}\right.\right.\left.,\left(\frac{r}{2} \frac{\partial V}{\partial r}+V\right) \psi_{\lambda}\right\rangle_{R}+\left(\frac{\alpha}{2}-1\right)\left\langle\psi_{\lambda}, V \psi_{\lambda}\right\rangle_{R} \mid \\
&=\left|\left\langle\psi_{\lambda}, V\left(\frac{r}{2 V} \frac{\partial V}{\partial r}+\frac{\alpha}{2}\right) \psi_{\lambda}\right\rangle_{R}\right| \leq \frac{\epsilon}{2}\left|\left\langle\psi_{\lambda}, V \psi_{\lambda}\right\rangle_{R}\right| .
\end{aligned}
$$

Thus

$$
\begin{aligned}
1-\frac{\alpha}{2}-\frac{\epsilon}{2} & \leq \frac{\left\langle\psi_{\lambda},\left(\frac{1}{2} r(\partial V / \partial r)+V\right) \psi_{\lambda}\right\rangle_{R}}{\left\langle\psi_{\lambda}, V \psi_{\lambda}\right\rangle_{R}} \\
& <1-\frac{\alpha}{2}+\frac{\epsilon}{2}
\end{aligned}
$$

so that

$1-\frac{\alpha}{2}-\frac{\epsilon}{2} \leq \underline{\lim } \frac{G(\lambda)}{\lambda G^{\prime}(\lambda)} \leq \varlimsup \lim \frac{G(\lambda)}{\lambda G^{\prime}(\lambda)} \leq 1-\frac{\alpha}{2}+\frac{\epsilon}{2}$.

Since $\epsilon$ is arbitrary,

$$
\lim \frac{G(\lambda)}{\lambda G^{\prime}(\lambda)}=1-\frac{\alpha}{2}=\frac{1}{\beta}
$$

so (i) is proved.

(ii) By Theorem 2.1, (e) implies (d) and (a) growth. Thus, by Theorems 5.3(a) and 5.5(a), if $V$ has attractive growth $\alpha(\mathrm{e})$, then

$$
G(\lambda) \sim \lambda^{\beta}(\mathrm{d})+(\mathrm{a}) .
$$

But (d) + (a) $\rightarrow$ (e) so $G(\lambda) \sim \lambda^{\beta}($ e). Moreover,

$$
\begin{aligned}
\lim _{\lambda \rightarrow \infty} \frac{G^{\prime}(\lambda)}{\lambda^{\beta-1}} & =\lim _{\lambda \rightarrow \infty} \frac{\lambda G^{\prime}(\lambda)}{G(\lambda)} \lim _{\lambda \rightarrow \infty} \frac{G(\lambda)}{\lambda^{\beta}} \\
& =\beta R_{\alpha} \lim _{r \rightarrow \infty} \frac{V(r)}{r^{\alpha}} \quad \text { (by } \\
& =\beta R_{\alpha}\left(\lim _{r \rightarrow 0} \frac{V^{\prime}(r)}{r^{\alpha-1}}\right)\left(\lim _{r \rightarrow 0} \frac{V(r)}{r V^{\prime}(r)}\right) \\
& =\frac{\beta R_{\alpha}}{\alpha} \lim _{r \rightarrow 0} \frac{V^{\prime}(r)}{r^{\alpha-1}} .
\end{aligned}
$$

\section{A CLASS OF EXAMPLES WITHOUT POWER GROWTH}

In this final section we construct potentials for which $G(\lambda)$ does not have power growth. On the basis of Sec. 5, we should try to construct them from potentials $V$ which do not have power growth at $\mathbf{r}=0$. In fact, we use central potentials which alternately grow as $r^{-\alpha_{1}}$ and $r^{-\alpha_{2}}$. The key to handling $G(\lambda)$ in this case is the following lemma:

Lemma 6.1: Let $V$ be a central potential which monotonically decreases as $r$ decreases. There is a positive constant $C$, independent of $V$, so that for any fixed $R$ and $\lambda$

$$
-\left(\frac{C}{R^{2}}+\lambda V(R)\right) \leq G(\lambda) \leq-\min _{r>0}\left[\frac{1}{4 r^{2}}+\lambda V(r)\right] .
$$

Proof: The upper bound follows from Theorem 4.5(i). To get the lower bound, pick (independently of $V$ ) some fixed normalized $C^{\infty}$ function $\phi$, with support inside the ball $\{\mathbf{r}|| \mathbf{r} \mid<1\}$ and let

$$
C=\langle\phi,-\Delta \phi\rangle\rangle 0 \text {. }
$$

Let $\phi_{R}(\mathbf{r})=R^{-\frac{3}{2}} \phi(\mathbf{r} / R)$, so that

$$
\int\left|\phi_{R}(r)\right|^{2} d^{3} r=1
$$

Then, by the variational principle, for any $R$,

$$
-G(\lambda) \leq\left\langle\phi_{R},(-\Delta+\lambda V) \phi_{R}\right\rangle .
$$

We have $\left\langle\phi_{R},-\Delta \phi_{R}\right\rangle=C / R^{2}$. Moreover, since $\operatorname{supp}\left(\phi_{R}\right) \subset\{\mathbf{r}|| \mathbf{r} \mid<R\}$ and $V$ is decreasing,

$$
\left\langle\phi_{R}, V \phi_{R}\right\rangle \leq V(R) \text {. }
$$

Thus $-G(\lambda) \leq\left(C / R^{2}\right)+\lambda V(R)$, which yields the lower bound.

Q.E.D.

We could have proven Theorem 5.3 using a strengthened form of Lemma 6.1.

We construct our examples by using the following basic fact about potentials which grow as a "variable power":

Lemma 6.2: Let $\gamma_{n}$ be a sequence of numbers with $0<\alpha_{1}<\gamma_{n} \leq \alpha_{2}<\frac{3}{2}$. Let $A_{n}$ be a sequence of numbers with

$0<\cdots<A_{n}<A_{n-1}<\cdots<A_{1}=1<A_{0}=\infty$.

Let $V(r)$ be given by

$$
V(r)=-r^{-\gamma_{n}}+B_{n},
$$

if $A_{n+1}<r<A_{n}$, where the $B_{n}$ 's are defined by $B_{0}=$ 0 , and by the requirement that $V$ be continuous. Let

$$
\delta_{n}=2 /\left(2-\gamma_{n}\right) \text {. }
$$

Then there exist constants $C_{1}$ and $C_{2}$ dependent only on $\alpha_{1}$ and $\alpha_{2}$, so that

$$
-\lambda B_{n}+C_{1} \lambda^{\delta_{n}}<G(\lambda)<-\lambda B_{n}+C_{2} \lambda^{\delta_{n}}
$$

whenever

$$
\frac{1}{2} \alpha_{1}^{-1}\left(A_{n}\right)^{\alpha_{1}-2}<\lambda<\left\{4\left[\left(A_{n+1}\right)^{2-\gamma_{n}}-B_{n}\left(A_{n+1}\right)^{2}\right]\right\}^{-1} .
$$

Proof: We first show that under the conditions on $\lambda$, that $\lambda V+4 r^{-2}$ takes its minimum value when $r \in\left(A_{n+1}, A_{n}\right)$. For suppose that $r>A_{n}$. Then, for any $m$,

$$
\gamma_{m} \lambda>\alpha_{1} \lambda>\frac{1}{2}\left(A_{n}\right)^{\left(\gamma_{1}-2\right)}>\frac{1}{2}\left(A_{n}\right)^{\alpha_{m}-2}>\frac{1}{2} r^{\gamma_{m}-2}
$$


(we have used the facts, $\alpha_{1} \leq \gamma_{m}<2$ and $A_{n}<1$ ). Thus

$(d / d r)\left(-\lambda r^{-\gamma_{m}}+\frac{1}{4} r^{-2}\right)=\left(\lambda \gamma_{m}-\frac{1}{2} r^{\gamma_{m}-2}\right) r^{-\gamma_{m}-1} \geq 0$,

so that $\lambda V+4 r^{-2}$ decreases monotonically in the region $r>A_{n}$, and so the minimum is taken for $r \leq A_{n}$.

On the other hand, the upper bound for $\lambda$ implies that

$$
\begin{aligned}
& \frac{-\lambda}{\left(A_{n+1}\right)^{\gamma_{n}}}+\lambda B_{n}+\frac{1}{4\left(A_{n+1}\right)^{2}} \\
& \quad=\frac{1}{4\left(A_{n+1}\right)^{2}}\left\{1-4 \lambda\left[\left(A_{n+1}\right)^{2-\gamma_{n}}-B_{n}\left(A_{n+1}\right)^{2}\right]\right\} \geq 0,
\end{aligned}
$$

so that $+\lambda V+\frac{1}{4} r^{-2} \geq 0$ at $r=A_{n+1}$. But $\left|r^{2} V\right|$ is monotone decreasing as $r \rightarrow 0$, so $\lambda V+\frac{1}{4} r^{-2}>0$ if $r<A_{n+1}$. Thus, we have shown that $\lambda V+4 r^{-2}$ takes its minimum value in $\left(A_{n+1}, A_{n}\right)$ and hence at the point

At this point

$$
r=R_{n}(\lambda) \equiv\left(\gamma_{n} \lambda\right)^{1 / \gamma_{n}-2}
$$

$$
\lambda V\left(R_{n}\right)=\lambda B_{n}-\left(\gamma_{n}\right)^{1 /\left(2-\gamma_{n}\right)} \lambda^{\delta_{n}}
$$

and

$$
R_{n}^{-2}=\left(\gamma_{n}\right)^{2 /\left(2-\gamma_{n}\right)} \lambda^{\delta_{n}} .
$$

Thus, using Lemma 6.1 with $R=R_{n}$, we see that

$$
-\lambda B_{n}+C_{1} \lambda^{\delta_{n}}<G(\lambda)<-\lambda B_{n}+C_{2} \lambda^{\delta_{n}}
$$

where

$$
\begin{aligned}
& C_{2}=\max _{\alpha_{1}<\gamma<\alpha_{2}}\left\{(\gamma)^{1 /(2-\gamma)}-\frac{1}{4}(\gamma)^{2 /(2-\gamma)}\right\}, \\
& C_{1}=\min _{\alpha_{1}<\gamma<\alpha_{2}}\left\{(\gamma)^{1 /(2-\gamma)}-C(\gamma)^{2 /(2-\gamma)}\right\} .
\end{aligned}
$$

To assure that $C_{1}>0$, we restrict ourselves to $\alpha_{2}<\min \left(1, C^{-1}\right)$. This restriction is not essential; if we were to work harder, we could probably remove it. However, we only want examples of nonpower growth-we do not wish to examine it in detail-so we take the lazy way out.

Theorem 6.3: Let $\alpha_{1}, \alpha_{2}$ be two numbers with $0<\alpha_{1}<\alpha_{2}<\min \left(1, C^{-1}\right)$, and let $\beta_{i}=2 /\left(2-\alpha_{i}\right)$.
Then there is a Kato potential $V$ with

$$
\underline{\lim } \frac{\log G(\lambda V)}{\log \lambda} \leq \beta_{1} ; \quad \varlimsup \lim \frac{\log G(\lambda V)}{\log \lambda} \geq \beta_{2} .
$$

In particular, $G$ fails to have power growth at $\infty$.

Proof: Pick the sequence $\gamma_{n}$ as in Lemma 6.2 by $\gamma_{2 m}=\alpha_{2}, \gamma_{2 m+1}=\alpha_{1}$. We shortly pick the $A_{n}$ 's and $B_{n}$ 's recursively. Once we have done this, we define $V$ as in Lemma 6.2. For all $r, V>-r^{-\alpha_{2}}$. Thus $V$ is Kato. Let $A_{1}=1$ and suppose we have picked $A_{2}, \cdots, A_{n}$, and thus also $B_{1}, \cdots, B_{n}$. If we had

$$
-\lambda B_{n}+C_{1} \lambda^{\delta_{n}}<G(\lambda)<-\lambda B_{n}+C_{2} \lambda^{\delta_{n}}
$$

for all $\lambda>\left(2 \alpha_{1}\right)^{-1} A_{n}^{\alpha_{1}-2}$, then it would follow that

$$
\lim _{\lambda \rightarrow \infty} \frac{\log G(\lambda)}{\log \lambda}=\delta_{n}
$$

so we pick $\lambda_{n}>\left(2 \alpha_{1}\right) A_{n}^{\alpha_{1}-2}$, so that

$$
\left|\frac{\log \left(-\lambda B_{n}+C_{1} \lambda^{\delta_{n}}\right)}{\log \lambda}-\delta_{n}\right|<\frac{1}{n}, \quad \text { at } \lambda=\lambda_{n},
$$

and

$$
\left|\frac{\log \left(-\lambda B_{n}+C_{2} \lambda^{\delta_{n}}\right)}{\log \lambda}-\delta_{n}\right|<\frac{1}{n}, \quad \text { at } \lambda=\lambda_{n}
$$

( $C_{1}>0$ is crucial here). Now pick $A_{n+1}$, so that

and

$$
\left\{4\left[\left(A_{n+1}\right)^{2-\gamma_{n}}-B_{n}\left(A_{n+1}\right)^{2}\right]\right\}^{-1}>\lambda_{n}
$$

$$
A_{n+1}<A_{n} .
$$

This is always possible since

$$
4\left[\left(A_{n+1}\right)^{2-\gamma_{n}}-B_{n}\left(A_{n+1}\right)^{2}\right]^{-1} \rightarrow \infty
$$

as $A_{n+1} \rightarrow 0$. Determine $B_{n+1}$ and proceed to pick $A_{n+2}, \cdots$. The $V$ so constructed has the property that $\log G\left(\lambda_{2 n}\right) / \log \lambda_{2 n} \rightarrow \beta_{2}$ and

$$
\log G\left(\lambda_{2 n+1}\right) / \log \lambda_{2 n+1} \rightarrow \beta_{1} .
$$

\section{ACKNOWLEDGMENT}

The author would like to thank M. Reed for an enlightening conversation on the nonpower growth of convex functions. 